\title{
EXPERIMENTAL INVESTIGATION OF FLOW FIELD AROUND AN INCLINED SQUARE CYLINDER UNDER FORCED OSCILLATION
}

\author{
TONGMING ZHOU, XIAOFAN LOU \& YUCEN LU \\ Department of Civil, Environmental and Mining Engineering, \\ The University of Western Australia, Australia
}

\begin{abstract}
Flow structures in the wake of a square cylinder inclined with angles of $\alpha=0^{\circ}, 15^{\circ}, 30^{\circ}$ and $45^{\circ}$ undergoing forced oscillation are investigated experimentally using particle image velocimetry (PIV) at Keulegan-Carpenter (KC) numbers in the range of 5-30 to examine the performance of the Independent Principle (IP). For $\mathrm{KC}<6$, there is no vortex shedding from the cylinder for all angles and as a result, the IP is valid. For $\mathrm{KC}=8-20$, IP does not work satisfactorily because at large inclination angles, the shear layers are stretched and reattach to the upper and lower sides of the cylinder body for most of the time within one oscillating cycle. When $\mathrm{KC}$ is increased further to 25, the phenomenon of shear layer attachment when the cylinder is at the neutral position, as well as the significant shedding at the end of each half cycle, indicate a similar flow field for both vertical and inclined cylinders. The present investigation indicates that the IP is valid when $\mathrm{KC} \leq 6$ and $\mathrm{KC} \geq 20$ as it is analogous to a steady current.
\end{abstract}

Keywords: square cylinder, vortex-shedding, inclined square cylinder, Independent Principle.

\section{INTRODUCTION}

Knowledge of the hydrodynamics of flow in the wake of a bluff structure is important. This is because the wake characteristics are closely related with the hydrodynamic forces and vortex shedding frequency. These two aspects are important considerations in the design of long bluff structures. Examples of these structures can be found in offshore oil and gas industry, such as the risers and pipelines, the pipes on the vessels for the production (e.g. the Floating Production, Storage and Offloading system (FPSO) or FLNG) and the transport tanker; the undersea cables for telecommunication. For these structures, the sea currents or waves may not always approach them perpendicularly. Instead, in most cases, the axis of these structures is aligned at an angle rather than $90^{\circ}$ to the sea currents and waves. In these cases, the incoming velocity can be decomposed into two components. One is perpendicular to the cylinder axis and the other is parallel to the cylinder axis. While the component perpendicular to the cylinder axis is important to the vortex shedding phenomenon, the component parallel to the axis may have adverse effect on the formation of the vortices and hence the hydrodynamic forces.

For a circular cylinder inclined to the incoming steady flow, extensive results have been reported before. It is generally accepted that if the Strouhal number, St $\left(\equiv f_{0} D / U_{\infty}\right)$, is normalized by the velocity component normal to the cylinder axis, i.e. $U_{N} \equiv U_{\infty} \cos (\alpha)$, its values should be comparable with that obtained in a cross flow. In the above relations, $f_{0}, D$ and $U_{\infty}$ are the vortex shedding frequency, diameter of the cylinder and freestream velocity, respectively. Analogously, if the drag coefficient $C_{D}\left(\equiv F_{D} /\left(1 / 2 \rho U_{\infty}^{2} D\right)\right.$ is normalized by velocity component normal to the cylinder axis, its values should be comparable with that obtained in a cross-flow. In this expression, $F_{D}$ is the drag force experienced by the cylinder in the fluid flow and $\rho$ is the fluid density. The above method to obtain the Strouhal number and the drag coefficient is generally termed as the Independent Principle (IP). 
For an inclined circular cylinder under forced oscillation or in waves, a number of studies have been reported in terms of force coefficients and vortex shedding frequency, e.g. [1], [2], in order to evaluate the IP in these flows. The flow and force characteristics in waves depends on both Reynolds numbers $\operatorname{Re}\left(\equiv U_{m} D / v\right.$, where $U_{m}$ is the maximum velocity of the sinusoidal oscillation and $v$ is the kinematic viscosity of the fluid) and Keulegan-Carpenter number $\mathrm{KC}$ ( $\equiv U_{m} T / D$, where $T$ is the oscillatory period). The combination of the above two parameters, i.e. Re and KC, is termed as the Stokes number $\beta$ (三 Re/KC) [3], [4]. Sarpkaya [1] showed that IP may not be valid for oscillatory flows, except for $\mathrm{KC}>20$, as at this condition, the oscillatory flow is similar to a steady current. He also argued that the Independent Principle is not valid in waves [1].

In engineering applications, the structures do not necessarily have a circular cross-section. A large amount of structures with a square or a rectangular cross-section are also used extensively. For these kinds of structures, the flow separation points are normally fixed at the sharp edge. Therefore, the flow and force characteristics of these kinds of structures reveal apparent dependence on the geometry of the cross-section [5], [6]. Whereas the validity of IP of a circular cylinder have been studied previously by a number of researchers (e.g. [7][11]) that of an inclined square cylinder is very limited. In a previous study by this research group [7], the validity of IP of an inclined square cylinder in steady flow has been evaluated at a Reynolds number of 7,000. In a more recent study by this group, the validity of IP of an inclined square cylinder in oscillatory flows was also examined in terms of the force coefficients and Strouhal number [12]. It was found that the IP for an inclined square cylinder in oscillatory flow is not valid for $\mathrm{KC}$ in the range of 8-20, at least when $\alpha$ is larger than $30^{\circ}$.

While the flow and hydrodynamic features of an inclined circular cylinder in oscillatory flows have been studied quite extensively, our knowledge about the wake characteristics of an inclined square cylinder in oscillatory flows is very limited. In the present study, the flow field of a square cylinder undergoing forced sinusoidal oscillation in quiescent water was studied at four different yaw angles, namely, $\alpha=0^{\circ}, 15^{\circ}, 30^{\circ}$ and $45^{\circ}$. Hereafter, the yaw angle $\alpha$ is defined as the angle between the flow direction and the cylinder axis (Fig. 1). However, in this paper, only flow fields for $\alpha=0^{\circ}$ and $45^{\circ}$ will be shown. This study aims to explore the mechanism for the invalidity of IP for $\mathrm{KC}$ in the range of $8-20$ [12]. The influence of the cylinder inclination angle on the formation, shedding and evolution of the vortices around the cylinder are studied by evaluating the phase-averaged vorticity patterns at different yaw angles and different $\mathrm{KC}$ numbers using PIV technique.

\section{EXPERIMENTAL SETUP}

\subsection{Oscillatory motion system}

The experimental setup used in the present study is shown in Fig. 1. The tests were conducted in a water flume of $0.4 \mathrm{~m}$ in width, $0.5 \mathrm{~m}$ in depth and $15 \mathrm{~m}$ in length. A belt-driven linear actuator (HISAKA) was used to achieve a linear oscillating movement along a long beam with amplitude $(A)$ in the range of $0-0.7 \mathrm{~m}$, i.e. $x(t)=A \sin (2 \pi t / \mathrm{T})(x$ is the position and is a function of time $t$ ). The solid cylinder rods with a side width of $10 \mathrm{~mm}$ and $20 \mathrm{~mm}$ made of clear acrylic material were cut with four different angles $\alpha=0^{\circ}, 15^{\circ}, 30^{\circ}$ and $45^{\circ}$ at both ends (Fig. 1). The ends of the cylinders were cut with their planes parallel to the flume bottom wall. A gap of $3 \mathrm{~mm}$ was left between the end of the cylinder and the bottom of the water flume to reduce the end effect. As the regime of the oscillatory flow around a cylinder is highly related to the $\mathrm{KC}$ numbers, the present study selected one amplitude to represent one flow regime within a certain $\mathrm{KC}$ range, e.g. $A=0.02 \mathrm{~m}$ corresponding to $\mathrm{KC}=6$ for no 


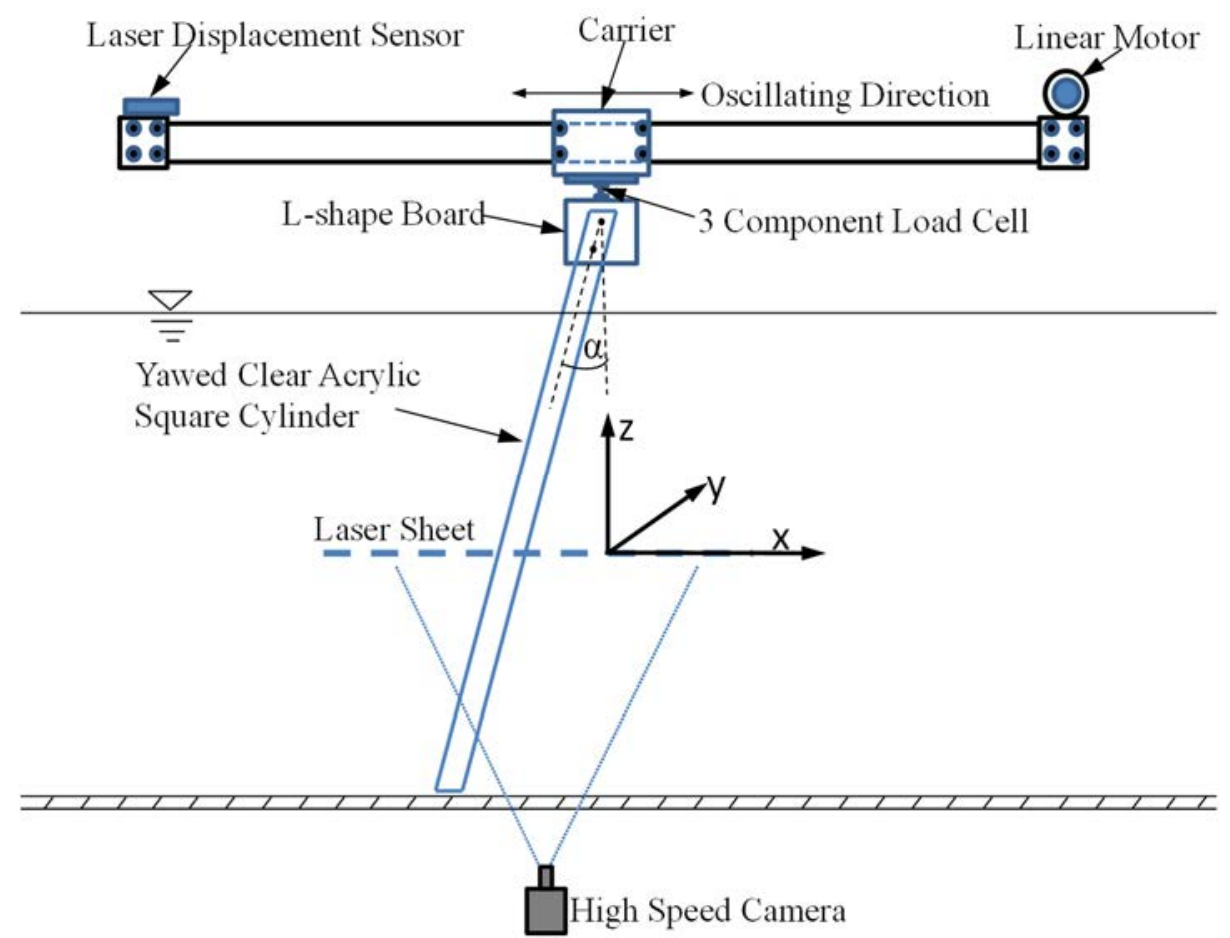

Figure 1: Schematic of the experimental setup (side view) and definition of the coordinate system.

Table 1: Experimental parameter at $2 \mathrm{~s}$ of oscillatory period at $\alpha=0^{\circ}$ for a square cylinder.

\begin{tabular}{|c|c|c|c|c|c|}
\hline $\mathrm{p}$ & Amplitude $(\mathrm{m})$ & $\mathrm{KC}$ & $R e$ & $\beta$ & Flow regime \\
\hline 0.02 & 0.02 & 6 & 1200 & 200 & No shedding \\
\hline 0.02 & 0.035 & 11 & 2200 & 200 & Single pair \\
\hline 0.01 & 0.03 & 19 & 1900 & 100 & Double pairs \\
\hline 0.01 & 0.04 & 25 & 2500 & 100 & Three pairs \\
\hline
\end{tabular}

vortex shedding regime, $A=0.035 \mathrm{~m}$ corresponding to $\mathrm{KC}=11$ for single pair of vortex regime, etc. Hydrodynamic forces were measured using a high sensitivity load cell. Table 1 lists the experimental parameters for the cases with $2 \mathrm{~s}$ of oscillatory period at $\alpha=0^{\circ}$.

\subsection{Flow visualization technique}

Flow fields were measured using a digital PIV system with a 5-watt continuous wave Argoion laser for illumination (Fig. 1). The laser sheet was about $1 \mathrm{~mm}$ in thickness and was aligned horizontally. The water was seeded with neutrally buoyant synthetic polycrystalline particles with a diameter of $1-5 \mu \mathrm{m}$. The images were captured through the glass window on 
the bottom wall of the flume using a high speed camera (Photron, FASTCAM SA3) with a resolution of 1024 pixels $\times 764$ pixels at a sampling rate of 1,000 frames/s. About 7,200 continuous flow fields, corresponding to about 15 oscillating cycles, were recorded for each case for the post-processing. The interrogation window size was 32 pixels $\times 32$ pixels with an overlap of $50 \%$, leading to an effective grid size of $3.2 \mathrm{~mm}$. About 2,800 vectors were generated for each image. The displacement of the cylinder was measured using a laser sensor. The displacement signals were used later as reference signals for the phase-averaging analysis using PIV.

\subsection{Force measurements}

The hydrodynamic forces on the cylinder were measured using a KISTLER load cell (Model 9317B), which was attached to the cylinder at the top end (Fig. 1). Details for the force measurements can be found in [12].

\section{RESULTS AND DISCUSSIONS}

\subsection{Force coefficients on the square and the circular cylinder}

Fig. 2 shows the drag coefficients of a circular and a square cylinder for $\alpha=0^{\circ}$ as an initial validation of the experimental setup. This figure was previously shown and discussed in [12]. It can be seen that the present drag coefficient reveals a satisfactory agreement with the previous experimental results at approximately the same $\beta$ numbers [13], [14] for a circular cylinder or at different $\beta$ numbers [6], [15] for a square cylinder. The agreement of the force coefficients at different $\beta$ values seems to support the conclusion obtained by Okajima et al. [15] that the drag coefficients of a square cylinder do not depend on $\beta$. The above results also indicate that the present experimental setup is reliable.

In Fig. 6 of the previously reported results [12], it has been shown that when $\mathrm{KC}<6$, IP is valid. However, for $\mathrm{KC}=8-20$, the departure of $C_{D N}$ at $\alpha=30^{\circ}$ and $45^{\circ}$ from that of $\alpha=$ $0^{\circ}$ is very apparent. The difference of $C_{D N}$ between $\alpha=0^{\circ}$ and $45^{\circ}$ can be as large as $44 \%$, which is too big to be attributed to experimental uncertainty, indicating that the IP is not valid when the cylinder inclination angle is large for $\mathrm{KC}=8-20$, at least when $\alpha \geq 30^{\circ}$. When $\mathrm{KC}$ is further increased to 20, the difference of $C_{D N}$ between $\alpha=0^{\circ}$ and $45^{\circ}$ is very small. This could be due to the reason that at these $\mathrm{KC}$ values, the flow approaches that of a steady current, at which condition, the IP is verified for $\alpha \leq 40-45^{\circ}$ [7], [16].

\subsection{Vortex pattern of a circular cylinder at $\mathrm{KC}=6$}

To verify the present experimental setup further, the instantaneous vorticity contours and the vector fields around a circular cylinder, corresponding to $\beta=200$ and $R e=1200$ at $\mathrm{KC}=6$, are shown in Fig. 3. Hereafter the dash lines represent negative contours rotating in clockwise direction and solid lines represent the positive contours rotating in counterclockwise direction. Both the $x$ and $y$ axes are normalized using the cylinder diameter $D$. The zero position along the $x$-axis denotes the neutral position of the oscillation and the zero position of the $y$-axis denotes the position of the centerline of the cylinder. Based on the classification of flow regimes from previous studies (e.g. [17]), the flow pattern is characterized by the generation of a pair of asymmetric vortices without shedding. Seeing from Fig. 3(a), a pair of vortices is formed on the right side of the cylinder when the cylinder 

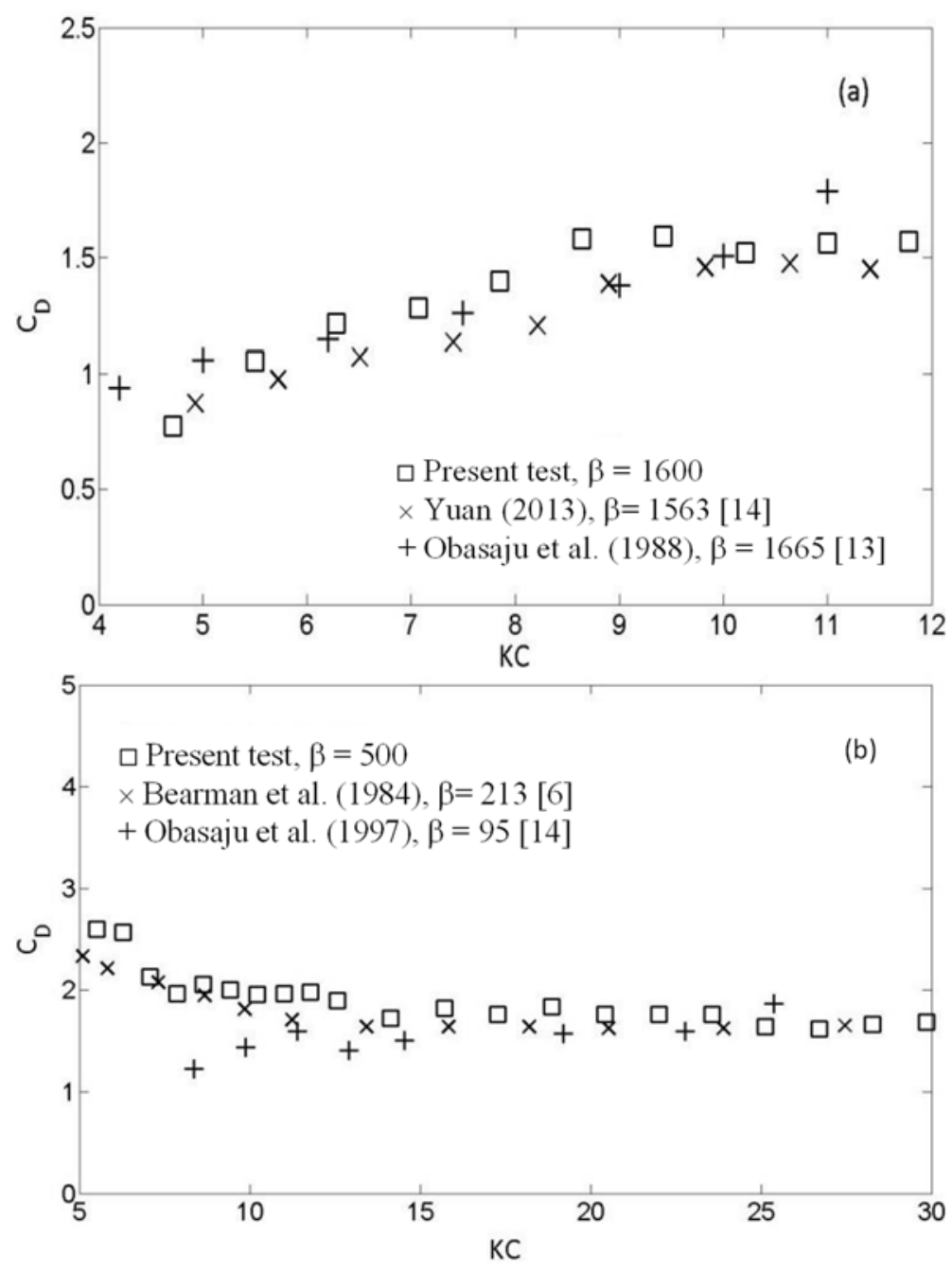

Figure 2: Comparison of drag coefficients with previous results for a circular cylinder and a square cylinder $\left(\alpha=0^{\circ}\right)$. (a) Circular cylinder; (b) Square cylinder.

just arrives at the leftmost position. As the cylinder is moving to the right, the vortex pair becomes asymmetric around the cylinder at $t=\mathrm{T} / 4$ when the negative vortex at the lower side of the cylinder dominates. In the second half cycle, similar process is observed except that the positive and negative vortices are swapped in locations. Clearly there is no shedding occurring as the attached vortex pair is washed away quickly over the cylinder surface and the detached vortices vanish rapidly. According to Lam et al. [18], no large-scale vortices can be completely shed from the cylinder when $\mathrm{KC}$ is smaller than 8 and only small vortices are found to remain attached to or detached for a short distance from the cylinder surface, which is similar to the present result. Both the vector fields displayed on the right hand column and the contour plots on the left hand column correspond well with the results for a circular cylinder at $\mathrm{KC}=6.28$ by Lam and Dai [19]. 

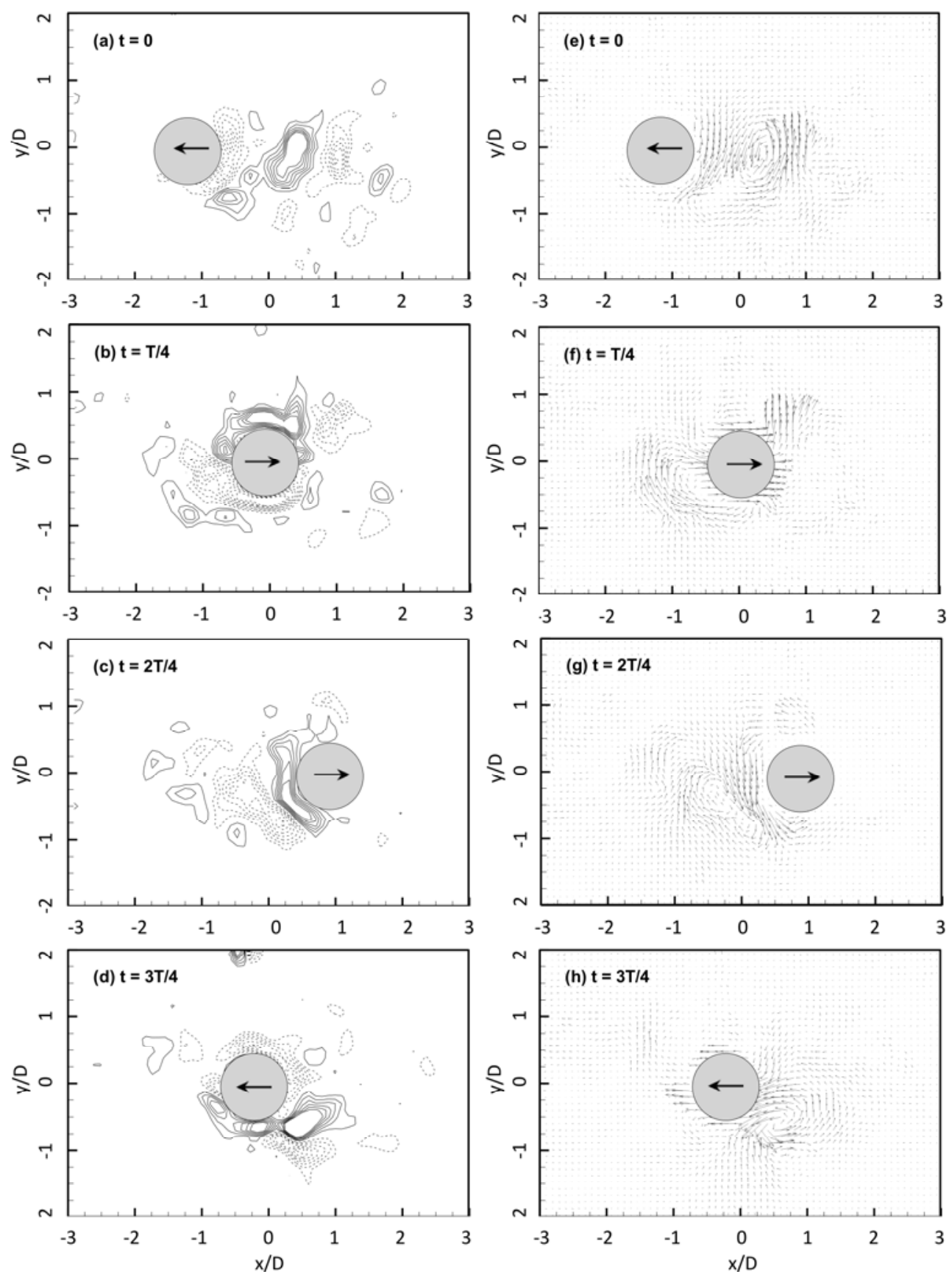

Figure 3: Instantaneous vorticity contours and corresponding vector fields around a circular cylinder within one cycle at $\mathrm{KC}=6 . \omega_{z \max }^{*}=7.3, \omega_{\text {zmin }}^{*}=-7.6$ and contour interval $=1$. 


\subsection{Vortex patterns at $\mathrm{KC}=11$ for $\alpha=0^{\circ}$ and $45^{\circ}$}

The results reported in [12] showed that for $\mathrm{KC}$ in the range of 8-20, the IP cannot be used to evaluate the force coefficient and vortex shedding frequency for an inclined square cylinder. It is expected that this phenomenon should be related with the change of vortex structures at large cylinder inclination angles. To explore the mechanism that $C_{D N}$ departs from IP over this $\mathrm{KC}$ range, the vortex shedding patterns at $\mathrm{KC}=11$ are discussed by examining the contours of the phase-averaged spanwise vorticity $\omega_{z}(\equiv \partial v / \partial x-\partial u / \partial y$, where $u$ and $v$ are velocity components in the $x$ and $y$ directions) at these two angles. The phaseaveraged vorticity contours around the cylinder at $\mathrm{KC}=11$ are shown in Figs 4 and 5 for $\alpha$ $=0^{\circ}$ and $45^{\circ}$, respectively, for which the displacement of the cylinder measured by the laser sensor is used as the reference signal for phase-averaging. At $\alpha=0^{\circ}$ and starting from $t=$ T/8 in Fig. 4(b), a pair of vortices with opposite signs is formed at either side of the cylinder. As the cylinder continues moving to the right, the positive vortex at the upper side grows faster than the negative one at the lower side. This asymmetric flow field leads to the generation of lift forces. From $t=2 \mathrm{~T} / 8$ to 3T/8, the positive vortex continues to stretch and wrap the negative one, displaying a trend to be shed. At the end of the first half cycle (Fig. 4(e)), the positive vortex is totally detached from the upper side of the cylinder. Meanwhile the negative vortex seems to shed from the lower side and moves along the left side of the cylinder to the upper side. One can also see another positive vortex starts to form at the lower side. Later the negative vortex is totally reattached to the upper side as the cylinder is moving from the rightmost position to the left at $t=5 \mathrm{~T} / 8$. The pair of vortices continues to grow and eventually sheds at the end of the cycle.

For cylinder inclination angle of $45^{\circ}$ (Fig. 5), the vortex structures are different from that of $0^{\circ}$. A pair of vortices just shed from the shear layers when the cylinder starts to move from the leftmost position to the right. What is more, the shear layers are stretched. From $t=\mathrm{T} / 8$ to $3 \mathrm{~T} / 8$, a pair of well-established vortices with similar strength is formed. At 3T/8, a vortex pair is shed from the leeward side of the cylinder (Fig. 5(d)). However, the newly shed vortex pair displays an asymmetric feature as there is only one well-established negative shedding contour observed when the cylinder reaches the rightmost position $(t=4 \mathrm{~T} / 8)$. When the cylinder starts to move from right to left, the negative vortex disappears fast as there is no vortex shedding or small-scale structure observed at the following phase. At the end of the cycle, a negative vortex is shed from the upper side of the cylinder while a positive vortex is about to shed but delayed by the stretched shear layer at the lower side. It can be seen that the vortex patterns at $\alpha=45^{\circ}$ are larger and stronger than that at $\alpha=0^{\circ}$. These vortex features could result in larger drag coefficient at $\alpha=45^{\circ}$ than that at $\alpha=0^{\circ}$, leading to the invalidity of the IP for $\mathrm{KC}=11$.

\section{CONCLUSIONS}

The hydrodynamic characteristics of flow around a square cylinder under forced oscillation at yaw angles of $0^{\circ}, 15^{\circ}, 30^{\circ}$ and $45^{\circ}$ were investigated using a PIV system and load cell to examine the validity of IP. The differences of vortex formation and vortex shedding at $0^{\circ}$ and $45^{\circ}$ were compared to illustrate the effect of the yaw angles on hydrodynamic forces and flow fields. At $\mathrm{KC}=6$, IP is valid. This result is due to the similar vortex characteristics between the vertical and inclined square cylinders. For $\mathrm{KC}=11$ and $\alpha=45^{\circ}$, the shear layers are stretched and reattached to the upper and lower surfaces of the cylinder for most time within one oscillating cycle. Therefore, the hydrodynamic behavior displays significant differences between the inclined and the vertical cylinders, leading to the invalidity of IP at this $\mathrm{KC}$ value. 

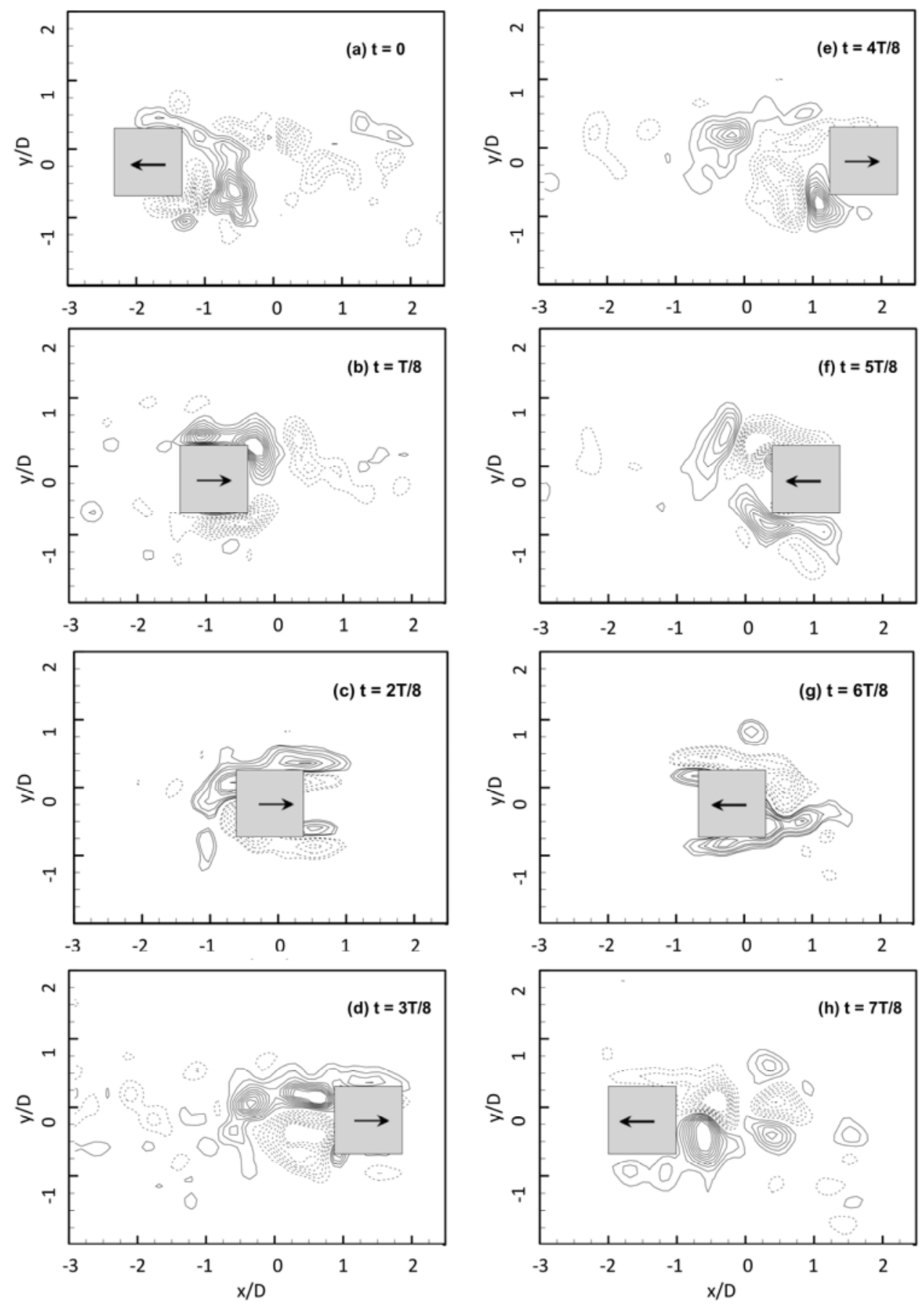

Figure 4: Phase-averaged spanwise vorticity contours around the square cylinder within one cycle at $\alpha=0^{\circ}$ and $\mathrm{KC}=11 . \omega_{z \max }^{*}=7.3, \omega_{z \min }^{*}=-6.5$ and contour interval $=1$. 

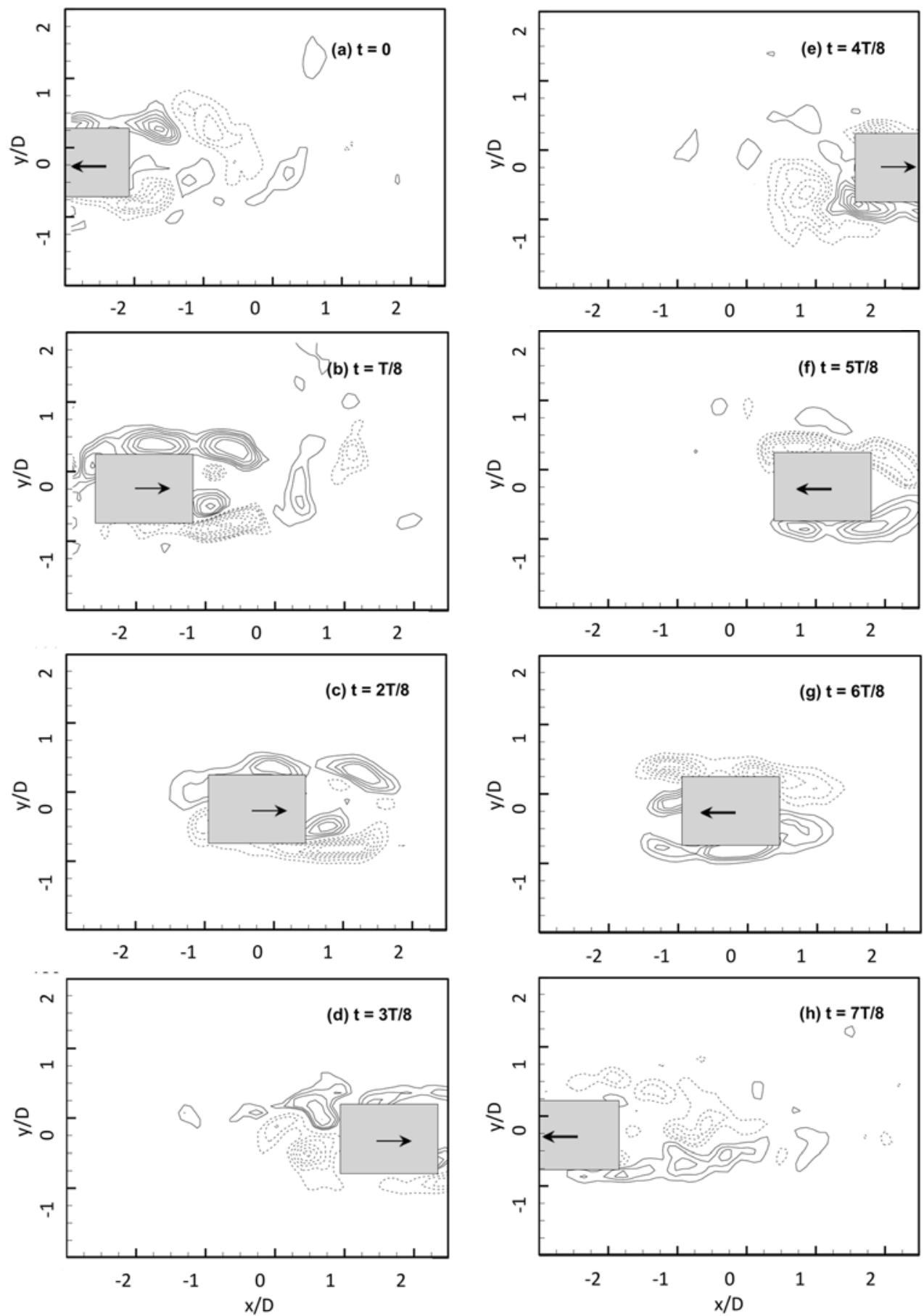

Figure 5: Phase-averaged spanwise vorticity contours around the square cylinder within one cycle at $\alpha=45^{\circ}$ and $\mathrm{KC}=11 . \omega_{z \max }^{*}=6.7, \omega_{\text {zmin }}^{*}=-6.2$ and contour interval $=1$. 


\section{REFERENCES}

[1] Sarpkaya, T., Wave forces on inclined smooth and rough circular cylinders. 14th Annual Offshore Technology Conference (OTC4227), Houston, Texas, 1982.

[2] Cotter, D. \& Chakrabarti, S., Wave force tests on vertical and inclined cylinders. $J$. Waterway, Port, Coastal and Ocean Engineering, 110, p. 18599, 1984.

[3] Chakrabarti, S.K., Cotter, D.C. \& Libby, A.R., Hydrodynamic coefficients of a harmonically oscillated tower. Applied Ocean Research, 5, pp. 226-233, 1983.

[4] Sundar, V., Vengatesan, V., Anandkumar, G. \& Schlenkhoff, A., Hydrodynamic coefficients for inclined cylinders. Ocean Engineering, 25, pp. 277-294, 1998.

[5] Bearman, P.W. \& Obasaju, E.D., An experimental study of pressure fluctuations on fixed and oscillating square-section cylinders. J. Fluid Mech., 119, pp. 297-321, 1982.

[6] Bearman, P.W., Graham, J.M.R., Obasaju, E.D. \& Drossopoulos, G.M., The influence of corner radius on the forces experienced by cylindrical bluff bodies in oscillatory flow. Applied Ocean Research, 6, pp. 83-89, 1984.

[7] Lou, X., Zhou, T., Wang, H. \& Cheng, L., Experimental investigation on wake characteristics behind a yawed square cylinder. Journal of Fluids and Structures, 61, pp. 274-294, 2016.

[8] Surry, D. \& Surry J., The effect of inclination on the Strouhal number and other wake properties of circular cylinders at subcritical Reynolds numbers. Technical Report, UTIAS Technical Institute for Aerospace Studies, University of Toronto, 1967.

[9] Ramberg, S.E., The effect of yaw and finite length upon the vortex wakes of stationary and vibrating circular cylinders. J. of Fluid Mech., 128, pp. 81-107, 1983.

[10] Thakur, A., Liu, X. \& Marshall, J.S., Wake flow of single and multiple yawed cylinders. J. of Fluids Engineering, 126, pp. 861-870, 2004.

[11] Marshall, J.S., Wake dynamic of a yawed cylinder. J. of Fluids Engineering, 125, pp. 97-103, 2003.

[12] Lou, X., Zhou, T., Wang, H. \& Cheng, L., Hydrodynamic coefficients of a yawed square cylinder in oscillatory. Ocean Engineering, 130, pp. 510-522, 2016.

[13] Obasaju, E.D., Bearman, P.W. \& Graham, J.M.R., A study of forces, circulation and vortex patterns around a circular cylinder in oscillating flow. J. Fluid Mech., 196, pp. 467-494, 1988.

[14] Yuan, Z., Experimental study of hydrodynamic forces on a surging circular cylinder in still water and in regular waves. PhD thesis, Nanyang Technological University, Singapore, 2013.

[15] Okajima, A., Matsumoto, T. \& Kimura, S., Force measurements and flow visualization of bluff bodies in oscillatory flow. J. Wind Engineering and Industrial Aerodynamics, 69-71, pp. 213-228, 1997.

[16] Zhou, T., Razali, S.F., Zhou, Y., Chua, L.P. \& Cheng, L., Dependence of the wake on yaw of a stationary cylinder. Experiments in Fluids, 46, pp. 1125-1138, 2009.

[17] Williamson, H.K., Sinusoidal flow relative to circular cylinders. Journal of Fluid and Mechanics, 155, pp. 141-174, 1985.

[18] Lam, K.M., Liu, P. \& Hu, J.C., Combined action of transverse oscillations and uniform cross-flow on vortex formation and pattern of a circular cylinder. Journal of Fluids and Structures, 26, pp. 703-721, 2010.

[19] Lam, K.M. \& Dai, G.Q., Formation of vortex street and vortex pair from a circular cylinder oscillating in water. Experimental Thermal and Fluid Science, 26, pp. 901915, 2002. 